\title{
Non-Hodgkin's Lymphoma: What Matters Tumor Type or Burden?
}

\author{
Journal of Postgraduate Medicine, Education and Research (2020): 10.5005/jp-journals-10028-1360 \\ CPC Editor \\ Pathology Discussant \\ Clinical Discussant \\ Radiology Discussant \\ Senior Resident \\ Clinician incharge \\ CPC Chairperson \\ DOA \\ DOD \\ Date of CPC \\ : Prof Ritambhra Nada ${ }^{1}$ \\ : Dr Balamurugan Thirunavukkarasu² \\ : Dr Ashish Behera ${ }^{3}$ \\ : Dr Nidhi Prabhakar ${ }^{4}$ \\ : Dr Divya Aggarwal ${ }^{5}$ \\ : Dr Deepesh Lad ${ }^{6}$ \\ : Prof Rakesh Kochar ${ }^{7}$ \\ : 30/06/2019 \\ : 12/07/2019 \\ : 12 February, 2020
}

\section{Case Description}

A 56-year-old female presented with multiple neck swellings for past 1 month, which were progressively enlarging. This was associated with reduced appetite, feeling of persistent fatigue, and low-grade fever. Over the past 2 weeks, she started having multiple episodes of vomiting, dull-aching upper abdominal pain, high colored urine, and yellowish discoloration of eyes and skin. It was associated with reduced alertness and swelling of bilateral lower limbs and progressive breathlessness for past 2 weeks, which had progressed from MMRC grade zero to 4 over the past 2 days. There was no history of high-grade fever, pruritus, clay colored or black tarry stools, bleeding per rectum, skin rash, or bleeding from any site. There was no history of arthralgia, oral ulceration, reduced urine output, frothuria, hematuria, or facial puffiness. There was no history of orthopnea, paroxysmal nocturnal dyspnea, chest pain, cough, or expectoration. There was no significant past history of diabetes mellitus, hypertension, bronchial asthma, tuberculosis, or oral ulcers. There was no similar or significant family history.

\section{EXAMINATION}

Patient was drowsy but easily arousable. She was pale and icteric and had generalized lymphadenopathy with presence of $2.5 \times$ $2.5 \mathrm{~cm}$ right submandibular lymph node, $3 \times 2 \mathrm{~cm}$ supraclavicular lymph node, and $1 \times 1 \mathrm{~cm}$ sized right axillary lymph node. The lymph nodes were firm, mobile, and non-tender and did not show any signs of inflammation. Her blood pressure was $100 / 78 \mathrm{~mm} \mathrm{Hg}$ at admission with a pulse rate of 128 beats per minute and a respiratory rate of 32 breaths per minute. She was maintaining $\mathrm{O}_{2}$ saturation of $90 \%$ on oxygen support in the form of venture mask delivering $40 \%$ oxygen. There were no cardiac murmurs. Fine crackles were heard in bilateral infrascapular regions, more on the left side.

Liver was palpable $4 \mathrm{~cm}$ below right costal margin and was tender with sharp margins, indicating hepatomegaly. Splenomegaly

\footnotetext{
1,2,5 Departments of Histopathology, Postgraduate Institute of Medical Education and Research, Chandigarh, India

${ }^{3,6}$ Departments of Internal Medicine, Postgraduate Institute of Medical Education and Research, Chandigarh, India

${ }^{4}$ Departments of Radiology, Postgraduate Institute of Medical Education and Research, Chandigarh, India

${ }^{7}$ Departments of Gastroenterology, Postgraduate Institute of Medical Education and Research, Chandigarh, India
}

Corresponding Author: Ritambhra Nada, Department of Histopathology, Postgraduate Institute of Medical Education and Research, Chandigarh, India, Phone: +91 7087008143, e-mail: ritamduseja@yahoo.com

How to cite this article: Nada R, Thirunavukkarasu B, Behera A, et al. Non-Hodgkin's Lymphoma: What Matters Tumor Type or Burden? J Postgrad Med Edu Res 2020;54(2):69-76.

Source of support: Nil

Conflict of interest: None

was also present as non-tender spleen palpable $4 \mathrm{~cm}$ below left costal margin. There was no evidence of free fluid in abdomen.

\section{INVESTIGATIONS}

- ANA-Negative

- ANCA (MPO, PR3)-Negative

- hsCRP-90.2 (normal-0-10)

- G6PD-normal

- IgG for L. donovani-Negative

- Widal test-Negative

- Malaria Ag-Negative

- Dengue/NS1-Negative

- Brucella serology-Negative

- HIV: Negative

(-) The Author(s). 2020 Open Access This article is distributed under the terms of the Creative Commons Attribution 4.0 International License (https://creativecommons. org/licenses/by-nc/4.0/), which permits unrestricted use, distribution, and non-commercial reproduction in any medium, provided you give appropriate credit to the original author(s) and the source, provide a link to the Creative Commons license, and indicate if changes were made. The Creative Commons Public Domain Dedication waiver (http://creativecommons.org/publicdomain/zero/1.0/) applies to the data made available in this article, unless otherwise stated. 
- HBsAg: Negative

- HCV: Negative

- Anti HAV (IgM)-Negative

- Anti HEV (IgM)-Negative

- Beta 2 microglobulin-25.48 mg/dL (Normal-1.22-2.46)

- Plasma hemoglobin-Not raised

- Urine hemoglobin-Nil

- Serum protein electrophoresis-Normal pattern. No M-band seen.

- Immunofixation electrophoresis-IgA Lambda (bands faint in dilution 1:9), No M-band

- Dr Aleena-Ultrasound-guided fine needle aspiration cytology was done from supraclavicular lymph node. The smears were cellular and showed dispersed population of atypical lymphoid cells which were 1.5-2 times the size of mature lymphocytes. These cells have irregular nuclei, coarse chromatin, 1-2 prominent nucleoli, and scant cytoplasm. Background showed few mature lymphocytes. Cell block showed similar features. Overall features were of high-grade non-Hodgkin's lymphoma (NHL).

- DrNarender-Peripheral blood smears showed 82\% neutrophils, mild left shift with $2 \%$ myelocytes, and $8 \%$ atypical lymphoid cells. RBCs were normocytic normochromic to macrocytic RBCs. Platelets were reduced with presence of few large platelets. Bone marrow aspirate smears revealed $14 \%$ atypical cells which were 2-2.5 times the size of mature lymphocytes with high N/C ratio, clumped chromatin, $0-1$ conspicuous nucleoli, round to irregular nuclear membrane, and agranular basophilic scant to moderate amount of cytoplasm. Bone marrow biopsy showed moderately hypercellular marrow spaces with an overall cellularity of $60-70 \%$ with interstitial increase of atypical cells. Megakaryocytes were adequate. On flowcytometry, these atypical lymphoid cells were positive for CD20, CD79b, and CD19. They showed lambda restriction and were negative for CD5, CD10, CD23, and CD34. So a diagnosis of bone marrow involvement by lambda restricted B cell lymphoma was given.
- Ultrasound abdomen-Liver was enlarged $(16.7 \mathrm{~cm})$ homogenous parenchyma. There was no evidence of dilatation of intrahepatic biliary radicals. Portal vein was normal. Gall bladder was contracted with presence of multiple gallstones, largest measuring $24 \mathrm{~mm}$. CBD was normal. Both the kidneys were normal. Spleen was enlarged measuring $16.2 \times 8.2 \mathrm{~cm}$. No free fluid was noted in abdomen.

- DrNidhi-CECT chest and abdomen (done on 01.07.19) showed right submandibular and right lower cervical lymphadenopathy. Mediastinal lymph nodes were enlarged which were causing compression of left subclavian artery. Paratracheal, subcarinal, and right hilar lymphadenopathy was also present. These lymph nodes were uniformly homogenous, with no necrosis or calcifications. No pleural or pericardial effusion was noted. Few small nodules were seen on lung windows, few of which were calcified. There were patchy areas of atelectasis in right middle lobe, lingula and bilateral lower lobes. Abdominal sections showed hepatomegaly and splenomegaly. No focal lesions or IHBRD were seen in liver. Cholelithiasis was noted with thickening of gall bladder wall. Similar retroperitoneal lymph nodes were seen encasing the aorta and inferior vena cava. The radiological differentials were of lymphoma and tuberculosis (lymphoma $\gg$ tuberculosis).

- Chest X-ray (10.07.19) showed inhomogeneous opacities in bilateral mid zones and left lower zone and homogenous opacities in left lower zone, which were obscuring the left CP angle suggestive of left pleural effusion. Few reticular markings were noted in bilateral upper zones.

- NCCTPNS and chest (done on 11.07.19): Lymph nodes were similar to those seen previously, and in comparison to previous CECT, patient had developed bilateral moderate pleural effusion and increased areas of atelectasis. Few areas of interlobular septal thickening in bilateral upper lobes and peribronchovascular thickening were seen, suggestive of interstitial pulmonary edema. PNS scans showed bilateral maxillary sinusitis.

- Rest of investigations: Refer to Tables 1 to 4

Table 1: Hemogram findings

\begin{tabular}{|c|c|c|c|c|c|}
\hline Date & $30-06-2019$ & $03-07-2019$ & 06-07-2019 & 08-07-2019 & $11-07-2019$ \\
\hline $\mathrm{Hb}(\mathrm{g} \%)$ & 7.9 & 7.8 & 6.6 & 7.6 & 8.3 \\
\hline TLC & 11,700 & 15,800 & 19,900 & 17,700 & 13,100 \\
\hline Diff. count & $68 / 09 / 20 / 03$ & & ANC $=13,930$ & $\mathrm{ANC}=12,744$ & ANC $=10,480$ \\
\hline Plt. count & 54,000 & 96,000 & 106,000 & 139,000 & 86,000 \\
\hline ESR & 29 & & & & \\
\hline
\end{tabular}

Table 2: Biochemistry: laboratory values

\begin{tabular}{llllll}
\hline Date & $30-06-2019$ & $03-07-2019$ & $06-07-2019$ & $08-07-2019$ & $11-07-2019$ \\
\hline Na+/K+ & $133 / 3.5$ & $140 / 4.14$ & $136 / 3.54$ & $142 / 3.8$ & $136.5 / 3.4$ \\
Urea/Cr & $57 / 1.3$ & $\mathbf{9 5 / 2 . 3 7}$ & $\mathbf{1 4 2 / 4 . 0}$ & $\mathbf{1 3 4 / 3 . 0 3}$ & $\mathbf{7 8 . 7 / 2 . 4 4}$ \\
Bil (T/D) & $21 / 12$ & $22 / 17$ & $21 / 14$ & $24 / 16.6$ & $25.93 / 17.49$ \\
SGOT/SGPT/ALP & $\mathbf{9 1 / 4 2 / 2 5 2}$ & $\mathbf{9 2 / 6 5 / 2 7 9}$ & $\mathbf{1 4 8 / 7 4 / 5 0 0}$ & $\mathbf{1 1 7 / 5 6 / 6 3 2}$ & $\mathbf{1 2 0 . 3 / 7 0 . 2 / 6 6 5}$ \\
T.Pro/ALBUMIN & 3 & $6.3 / 3.23$ & $4.4 / 2.17$ & $5.9 / 3.2$ & $3.77 / 2.60$ \\
Mg+/Ca+2/PO4- & & $2.99 / 9.26 / 2.68$ & $2.74 / 9.49 / 3.2$ & $2.44 / 11.3 / 3.96$ & $3.18 / 9.65 / 3.85$ \\
LDH (135-225 U/L) & $\mathbf{4 3 4}$ & $\mathbf{3 1 8}$ & $\mathbf{3 1 2}$ & $\mathbf{1 0 4 4}$ & $\mathbf{4 4 7}$ \\
Chl/LDL/HDL/TG & & & $130 / 13.7 / 5 / 392$ & & \\
(mg/L) & & & & & \\
\hline
\end{tabular}


Non-Hodgkin's Lymphoma: What Matters Tumor Type or Burden?

Table 3: Coagulation profile

\begin{tabular}{|c|c|c|c|c|c|}
\hline Date & 30-06-2019 & 03-07-2019 & 07-07-2019 & 10-07-2019 & $11-07-2019$ \\
\hline PTTK/aPTT (28-35 seconds) & 30.5 & 33 & 38.2 & 36.4 & 50.7 \\
\hline PT (12-15 seconds) & 19.2 & 18 & 21 & 17.6 & 19.4 \\
\hline PTI (\%) & 72 & 74 & 66 & 76 & 69 \\
\hline INR & 1.37 & 1.29 & 1.57 & 1.26 & 1.39 \\
\hline $\begin{array}{l}\text { Fibrinogen }(2-4 \mathrm{~g} / \mathrm{L}) / \mathrm{D} \text {-dimer } \\
(0.5 \mu \mathrm{g} / \mathrm{mL})\end{array}$ & & $4.3 / 2$ & & $5.36 / 2.31$ & $1.14 / 4.88$ \\
\hline
\end{tabular}

Table 4: Arterial blood gas values

\begin{tabular}{|c|c|c|c|c|c|}
\hline Date & 30-06-2019 & 02-07-2019 & 06-07-2019 & 08-07-2019 & $11-07-2019$ \\
\hline $\mathrm{pH}$ & 7.539 & 7.47 & 7.36 & 7.342 & 7.153 \\
\hline $\mathrm{HCO}_{3}$ & 18.9 & 18 & 14.9 & 15.8 & 18.8 \\
\hline $\mathrm{SpO}_{2}$ & 84 & 68 & 69.7 & 52.3 & 85.7 \\
\hline $\mathrm{pCO}_{2}$ & 22.7 & 25 & 26.4 & 29.8 & 54.7 \\
\hline $\mathrm{SO}_{2}$ & 90.3 & 90 & 93.9 & 85.7 & 93.4 \\
\hline Lactate & 1.77 & 3.09 & 3.57 & 6.13 & 5 \\
\hline
\end{tabular}

\section{Course and Management}

This 56-year-old female was admitted with moderate anemia, thrombocytopenia, lymphadenopathy, hepatosplenomegaly, conjugated hyperbilirubinemia, hypoalbuminemia, and history of B symptoms for last 1 month. She presented with sepsis, metabolic acidosis, and hypoxemia and was managed with piperacillin/ tazobactam and supplemental oxygen with a provisional clinical diagnosis of cholangitis. She developed hypotension on next day, with deterioration of sensorium and was started on vasopressor support which was needed to be up-titrated to 3 drugs over the due course and noninvasive ventilation for the progressive respiratory distress. Negative consent was given for intubation and she was hence continued on noninvasive ventilation. She developed deterioration of sensorium, worsening metabolic acidosis, and acute kidney injury with oliguria.

She was treated with sustained low efficiency dialysis for the renal impairment starting from day 4 of admission (03.07.19). She developed increased infiltration of lung field along with refractory shock with elevation of lactate and procalcitonin for which her antibiotics were hiked up and caspofungin was started with a clinical suspicion of fungal sepsis.

Once a diagnosis of high-grade NHL was established using FNAC, she was started on dexamethasone, cyclophosphamide $\left(800 \mathrm{mg} / \mathrm{m}^{2}\right)$, and rituximab $\left(375 \mathrm{mg} / \mathrm{m}^{2}\right)$. She continued to have deterioration of vitals and renal function (anuria) in spite of the definitive treatment and supportive management. The patient succumbed to her illness and sustained cardiac arrest on 12.07.19.

Lymph node biopsy was done whose report was available postmortem. Lymph node biopsy showed effaced nodal architecture with presence of atypical cells in sheets, which were 2.5-3 times the size of mature lymphocytes. These atypical lymphoid cells were positive for CD20, Bcl2, and MUM1 (more than $30 \%$ cells) and were negative for CD3, CD10, cyclin D1, SOX 11, and Bcl6. The final diagnosis rendered was diffuse large $B$ cell lymphoma (DLBCL) and activated $B$ cell $(A B C)$ phenotype.

\section{Case Analysis}

A 56-year-old lady presented with fever, loss of appetite, lymphadenopathy, hepatosplenomegaly, deep jaundice,
Table 5: Factors of revised international prognostic index for DLBCL

\begin{tabular}{l} 
Age greater than 60 years \\
ECOG performance status greater than 2 \\
Elevated LDH (above normal range) \\
More than 1 extranodal site \\
Ann Arbor Stage III/IV \\
R-IPI score based on number of factors present \\
Very good \\
Good \\
Poor \\
\hline
\end{tabular}

deterioration of sensorium, shortness of breath, and refractory septic shock whose investigations showed leukocytosis, thrombocytopenia, conjugated hyperbilirubinemia, coagulopathy, metabolic acidosis, and hypoxemia. The basic disease was revealed on lymph node FNAC and bone marrow examination to be $D L B C L$, $A B C$ phenotype.

The ABC phenotype is classified as an aggressive NHL. Elevated beta-2 microglobulin levels render an unfavorable prognosis. ${ }^{2}$ In this patient, the DLBCL may have been of extranodal origin, which accounts for $40 \%$ of all DLBCLs or it may have been advanced DLBCL with spread to extranodal organs. ${ }^{3}$ As per revised international prognostic index for DLBCL (Table 5), this patient had a score of 4 with poor prognosis and estimated 4 -year survival of $44 \% .{ }^{4}$

The patient had accompanying acute liver failure which contributed to rapid deterioration, as indicated by jaundice, coagulopathy, hepatic encephalopathy, and no evidence of prior liver disease. The likely etiology of acute liver failure could be malignant infiltration of liver, secondary hemophagocytic lymphohistiocytosis (HLH), infective etiology, drug intake, and veno-occlusive disease. Malignant infiltration of liver is most likely as there is hepatomegaly without any focal liver lesion, with disproportionate elevation of ALP along with aggressive form of DLBCL. ${ }^{5}$ The $\mathrm{H}$ score was 188 , and hence, a possibility of hereditary lymphohistiocytoses (HLH) was also considered, though bone marrow did not show any hemophagocytosis. ${ }^{6}$

Breathlessness in the patient could be attributable to infiltration of lung by lymphoma or an infection (bacterial > fugal > viral) 
since the radiological features showed bilateral lung infiltrates and patient had presence of factors predisposing to infection, i.e., high-grade NHL.

The terminal event was refractory septic shock as the SOFA score was 18 with acute kidney injury and elevated lactate and procalcitonin. ${ }^{7}$ Additionally volume overload also likely played a role terminally, as the patient had anemia, hypoalbuminemia, renal failure, and there was evidence of pleural effusion and pulmonary edema on radiology.

\section{Final Clinical Diagnosis}

- B-cell non-Hodgkin's lymphoma- DLBCL stage IV E (ECOG-4) with bone marrow and liver infiltration

- Acute liver failure (MELD = 36) (coagulopathy and encephalopathy)

- Community acquired pneumonia? Aspiration pneumonia

- Candidemia

- Refractory septic shock

\section{Clinical Discussion}

Prof Rakesh Kochar: Thank you Dr Behera. Please join me here. So this patient was admitted under hematology services. If there is anyone from the unit to comment upon the clinical course, please come.

Dr Niranjan: Good morning. So this elderly female was admitted with us in the HDU with a stay of around 13 days. So as we can see, what is very clear is that we are dealing with a very aggressive kind of lymphoma. I am calling it aggressive because the presentation was short with severe organ failure; she had liver infiltration causing hepatic encephalopathy and coagulopathy as well as the other biological markers, the IPI was 4, and beta-2 microglobulin was high. LDH was quiet high and she also had hypercalcemia. So calcium of $11.9 \mathrm{mg} / \mathrm{dL}$ was the maximum calcium. There is lymphoma causing liver infiltration, some component of HLH and hemolysis cannot be ruled out. What can this aggressive lymphoma be? So we were thinking that this could be a double-hit or a triple-hit lymphoma with MYC translocations. Another consideration could be an underlying CLPD with Richter's transformation, since there was marrow infiltration with an $\mathrm{M}$ band, but the marrow morphology is going more in favor of an aggressive disease only. The terminal event, I think, is predominantly sepsis. The patient came to us with liver failure, we had given her dexamethasone, cyclophosphamide. She had severe septic shock. She had candidemia in blood. So I think the terminal event was predominantly sepsis in a patient who had an active disease due to chemotherapy.

Prof Rakesh Kochar: Thank you.

Dr Gaurav: So just to reiterate what Dr Niranjan has said, seeing atypical cells in the periphery in a patient with DLBCL is exceedingly rare, and with the extensive extranodal involvement, we are possibly dealing with a double-hit or a triple-hit lymphoma with MYC translocation. Even though on immunohistochemistry, it is $A B C$ subtype and we know that it is less likely. Second point is that the patient was hypoxic right to begin with. She needed high oxygen requirement at $40 \% \mathrm{FiO}_{2}$ and then maintaining saturation at $90 \%$. First CT was relatively okay with presence of focal atelectasis. So possibly there is some component of a pulmonary thromboembolism or possibly, I would say, there is some interstitial infiltration by the lymphoma into the lungs is possibly causing the hypoxia.
Prof Rakesh Kochar: Yes, Dr Virender Singh.

Prof Virender Singh: There are few important points There was no question of cholangitis in this patient. The ultrasound never showed biliary dilatation. And then, such a large hepatomegaly with the lymph nodes, I do not think there is any doubt, especially after the FNAC, the lymphoma is there. Then regarding the acute liver failure (ALF), this presentation cannot be labeled ALF. In ALF, there is a gross transaminitis; INR is deranged grossly which is absent here. Even when total bilirubin at the onset was 21 , the INR was 1.1 or 1.2 , and we never see in ALF. Further we never get raised ALP in ALF which was present in this patient. So this was a massive infiltration of the liver because of the lymphoma and I am not expecting a massive necrosis of the liver on the autopsy.

Prof Rakesh Kochar: Okay, with this we move on to the pathology protocol to be presented by Dr Balamurugan.

\section{Pathology Findings}

A partial autopsy was carried out. The prosectors noted that the deceased was moderately built. The pleural and pericardial cavities were within normal limits and the peritoneal cavity yielded $500 \mathrm{~mL}$ of straw colored fluid.

Lymph node biopsies from submandibular and mediastinal lymph nodes performed antemortem showed sheets of atypical lymphoid cells which were 2-3 times the size of mature lymphocytes with condensed chromatin and occasional prominent nucleoli (Fig. 1). They were immunopositive for CD20, Bcl2 (>70\%), and MUM-1 (>30\%). Ki67 labeling index was 40-50\% (Fig. 1).

Para-aortic and hilar lymph nodes were enlarged and showed effaced nodal architecture and replacement by sheets of atypical lymphoid cells with similar morphology. The cells were negative for CD3, CD5, CD30, CD10, Bcl6, c-myc, Tdt, Cyclin D1, SOX11, and EBER ISH (Fig. 2). Features were of a diffuse large B-cell lymphoma, non-GCB phenotype, and $\mathrm{BCl} 2$ expressor.

Interphase FISH (break-apart probe) performed for c-myc, Bcl2, and $\mathrm{Bcl} 6$ was negative (Fig. 2). Occasional necrotizing epithelioid cell granulomas were identified (Fig. 2). Ziehl-Neelsen stain for acid fast bacilli was negative. Mesenteric lymph nodes showed atypical lymphoid cells with similar morphology (Fig. 2).

Liver-weighed $3100 \mathrm{~g}$. The capsular surface was normal with no wrinkling or nodularity. Cut surface was mottled (Fig. 3). Biliary tree, portal, and hepatic veins were normal. Microscopy showed distorted lobular architecture. Subcapsular surface, portal tracts, and sinusoids showed diffuse, massive infiltration by similar atypical lymphoid cells (Fig. 3). DLBCL presenting as acute liver failure is rare though rare cases have been reported ${ }^{8,9}$

Spleen-weighed $650 \mathrm{~g}$. Capsular aspect was unremarkable while the cut surface was congested (Fig. 3). Microscopy showed diffuse infiltration of sinusoids by atypical lymphoid cells with similar morphology as described above (Fig. 4).

Bone marrow-was hypercellular for age (Fig. 4) and showed adequate representation of all three hematopoietic lineage elements. However, occasional clusters of atypical lymphoid cells were identified and these cells were positive for CD20 indicating infiltration by lymphoma (Fig. 4).

Lungs-Both lungs weighed $1140 \mathrm{~g}$ and were heavy (Fig. 5). Lungs were subcrepitant to feel. The pleura was shiny except over the left lower lobe of lung. Cut surface of this region in left lower lobe showed hemorrhagic consolidation (Fig. 5). Hilar and carinal lymph nodes measured $0.5-0.8 \mathrm{~cm}$ in diameter. No thrombus was seen in the major pulmonary vessels. Microscopic examination from the hemorrhagic lesions showed diffuse alveolar hemorrhage 


\section{A}
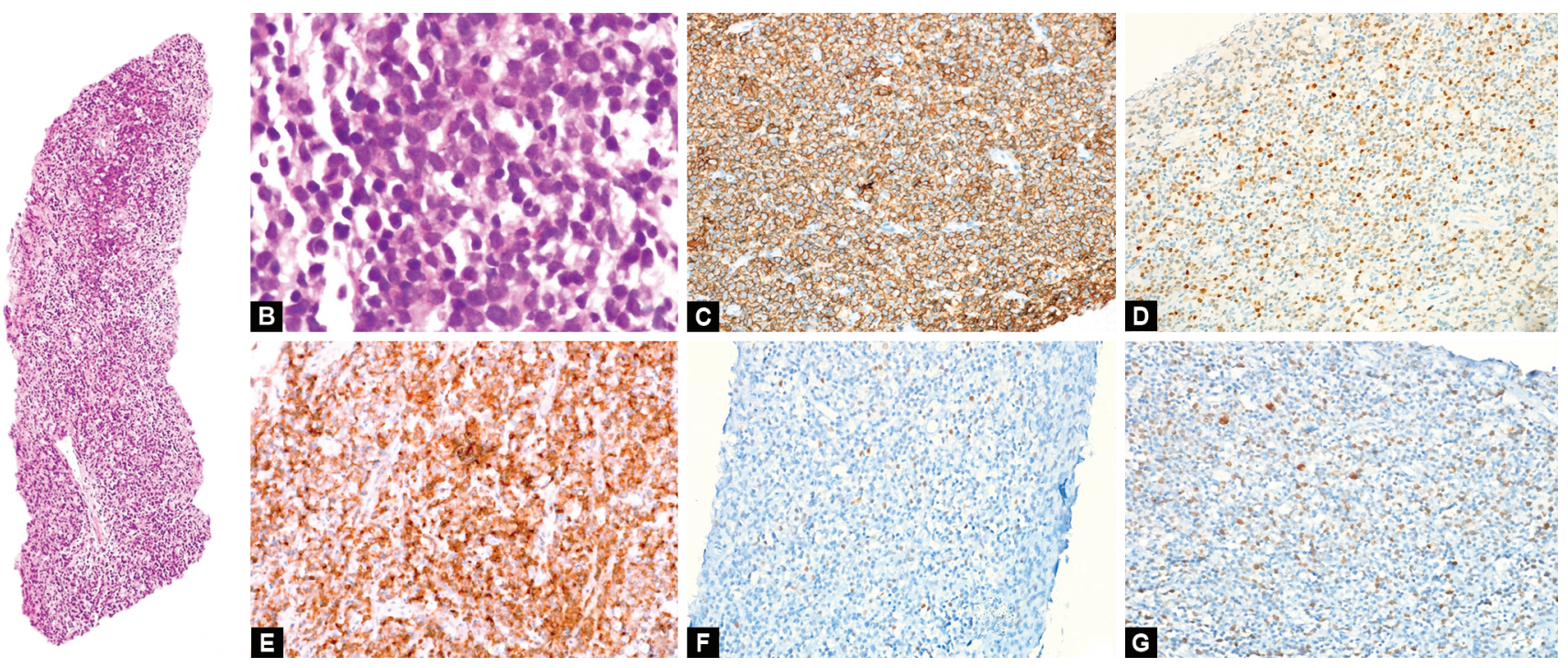

Figs 1 A to G: (A) Core biopsy showed diffuse sheets of atypical lymphoid cells (hematoxylin and eosin 40X); (B) These atypical lymphoid cells were 2-3 times the size of mature lymphocytes with condensed chromatin and occasional prominent nucleoli (hematoxylin and eosin 400X). They were positive for CD20 [(C) CD20 immunohistochemistry, 200x], Bcl2 (in >70\% cells); [(D) Bcl2 immunohistochemistry, 200×], MUM1 (in >30\% tumor cells); [(E) MUM1 immunohistochemistry, 200x], and negative for c-myc; [(F) c-myc immunohistochemistry, 200x]. Ki67 labeling index was 40\%-50\%; [(G) Ki67 immunohistochemistry, 200×]
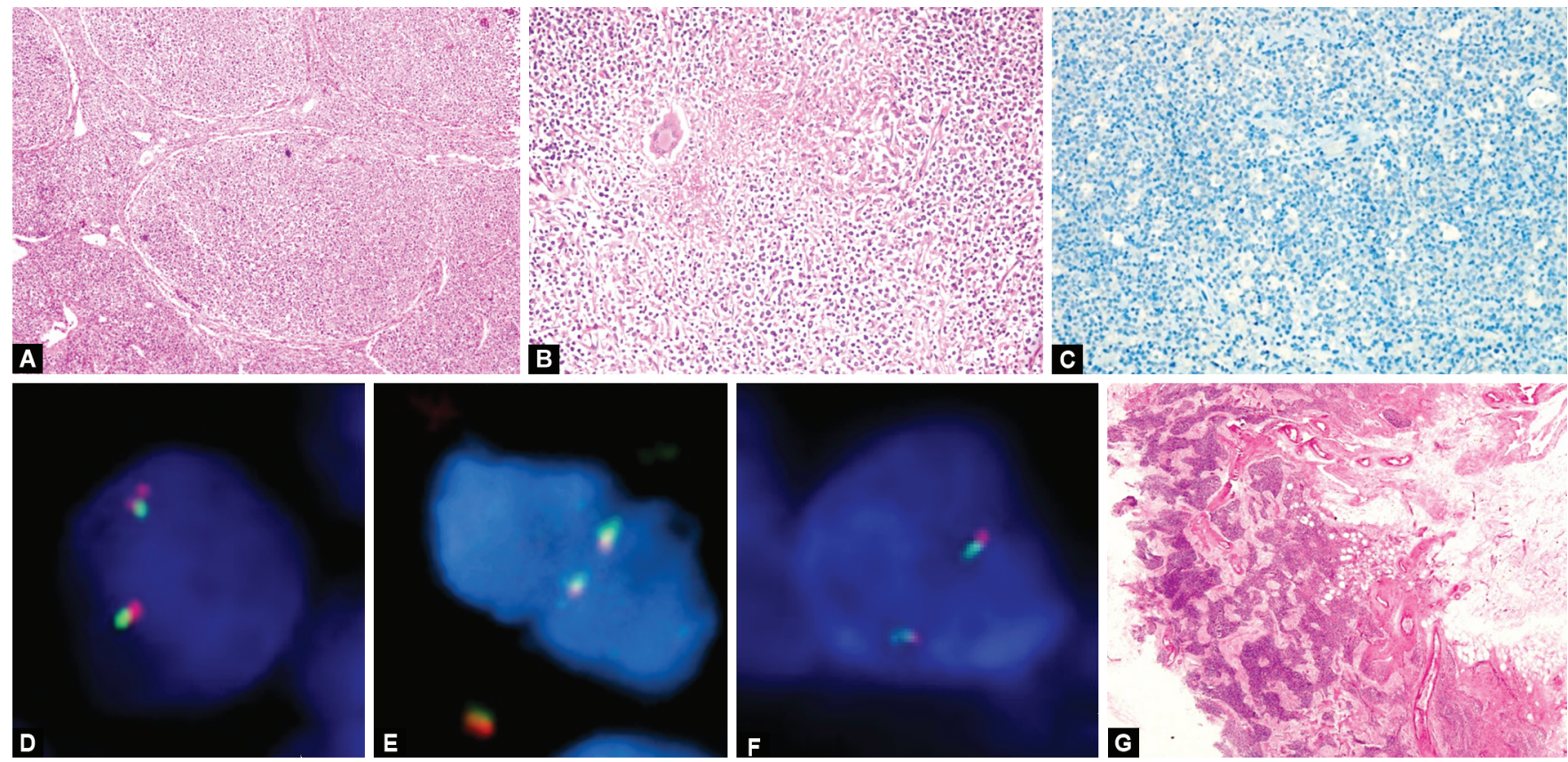

Figs 2A to G: (A) Mediastinal lymph node had effaced nodal architecture with replacement by sheets and large nodules of large atypical lymphoid cells (hematoxylin and eosin, 40X); (B) There were occasional epithelioid cell granulomas (hematoxylin and eosin 100X); (C) These atypical lymphoid cells were negative for EBER-ISH (EVER-ISH, 200X); (D) Merged image of FISH with dual colored break-apart probe for c-myc showing two normal fused signals (1000X, oil immersion); (E) Merged image of FISH with dual colored break-apart probe for Bcl2 showing two normal fused signals (1000x, oil immersion); (F) Merged image of FISH with dual colored break-apart probe for Bcl6 showing two normal fused signals (1000x, oil immersion); (G) Mesenteric lymph nodes showed infiltration by atypical lymphoid cells with similar morphology (hematoxylin and eosin 40X)

(Fig. 5). No fungal profiles, granulomas, or evidence of lymphoma infiltration was seen.

Heart-weighed $330 \mathrm{~g}$. Pericardium was unremarkable and all four chambers were grossly unremarkable (Fig. 5). The right ventricular wall thickness was $0.4 \mathrm{~cm}$ and left ventricular wall thickness was $1.4 \mathrm{~cm}$ (Fig. 5). All cardiac valves were within normal limits and no vegetations were noted. Aorta showed grade I atherosclerosis. Myocardium was microscopically unremarkable.

Kidneys-both kidneys weighed $260 \mathrm{~g}$. Capsular surface was unremarkable and capsule was easily stripped off. The cut surface showed distinct corticomedullary junction with medullary congestion. Microscopy showed acute tubular necrosis with bile 

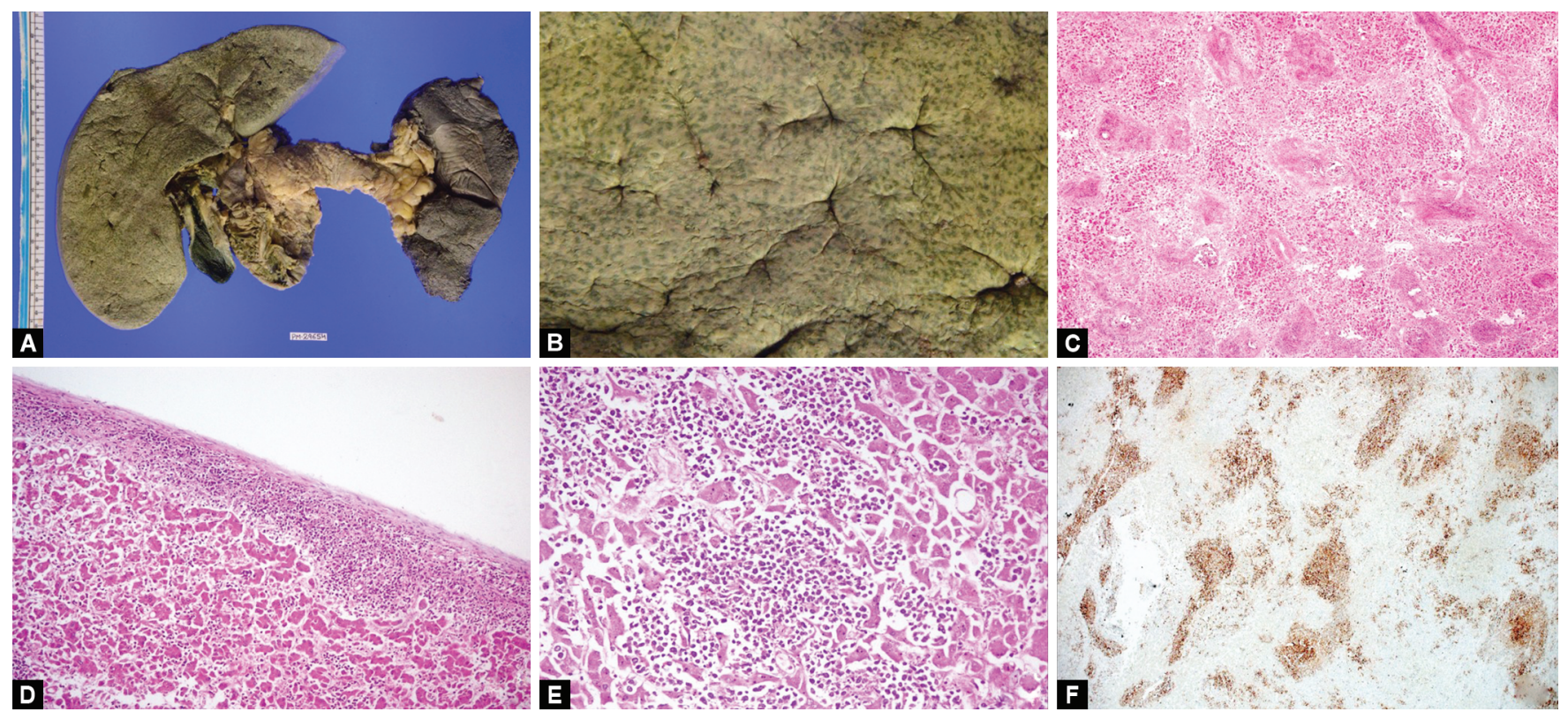

Figs 3A to F: (A) Gross photograph of organ complex comprising of slice of liver, gallbladder, C loop of duodenum, head of pancreas, and slice of spleen. The liver was grossly enlarged; (B) Cut surface of liver showed mottled appearance; (C) The architecture of liver was distorted and the portal tracts showed infiltration by atypical lymphoid cells (hematoxylin and eosin, 40x); (D) Subcapsular region of liver showed infiltration by similar cells (hematoxylin and eosin 100X); (E) Atypical lymphoid cells were also seen diffusely infiltrating the sinusoids (hematoxylin and eosin 200x); (F) These atypical lymphoid cells were positive for CD20 (CD20 immunohistochemistry, 40x)
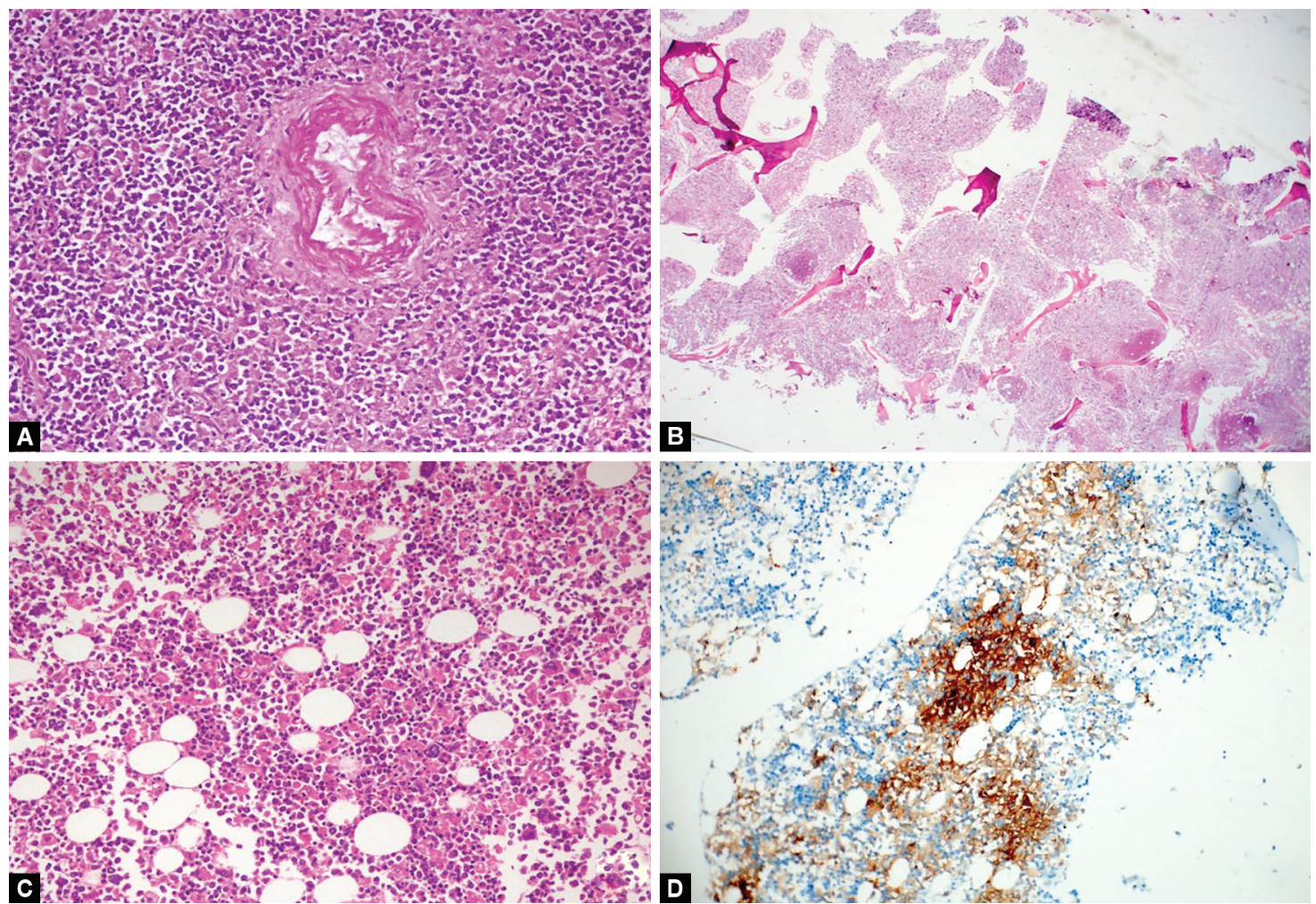

Figs 4A to D: (A) Spleen showed diffuse infiltration of sinusoids by atypical lymphoid cells (hematoxylin and eosin 200x); (B) Bone marrow was hypercellular for age (hematoxylin and eosin 20X); (C) Bone marrow showed infiltration by atypical lymphoid cells (hematoxylin and eosin 400x); (D) These atypical lymphoid cells were positive for CD20 (CD20 immunostain 100x)

cast nephropathy (Fig. 6). Glomeruli, interstitium, and blood vessels showed no significant pathology.

Uterus and ovaries-Endometrium was atrophic. An intramural fibroid measuring $4.5 \times 3 \mathrm{~cm}$ was identified in the fundus and body.
Microscopy showed features of benign leiomyoma. Bilateral ovaries were grossly and microscopically unremarkable.

Esophagus and stomach-Distal esophagus and cardia of stomach showed erosion with areas of hemorrhage which were 

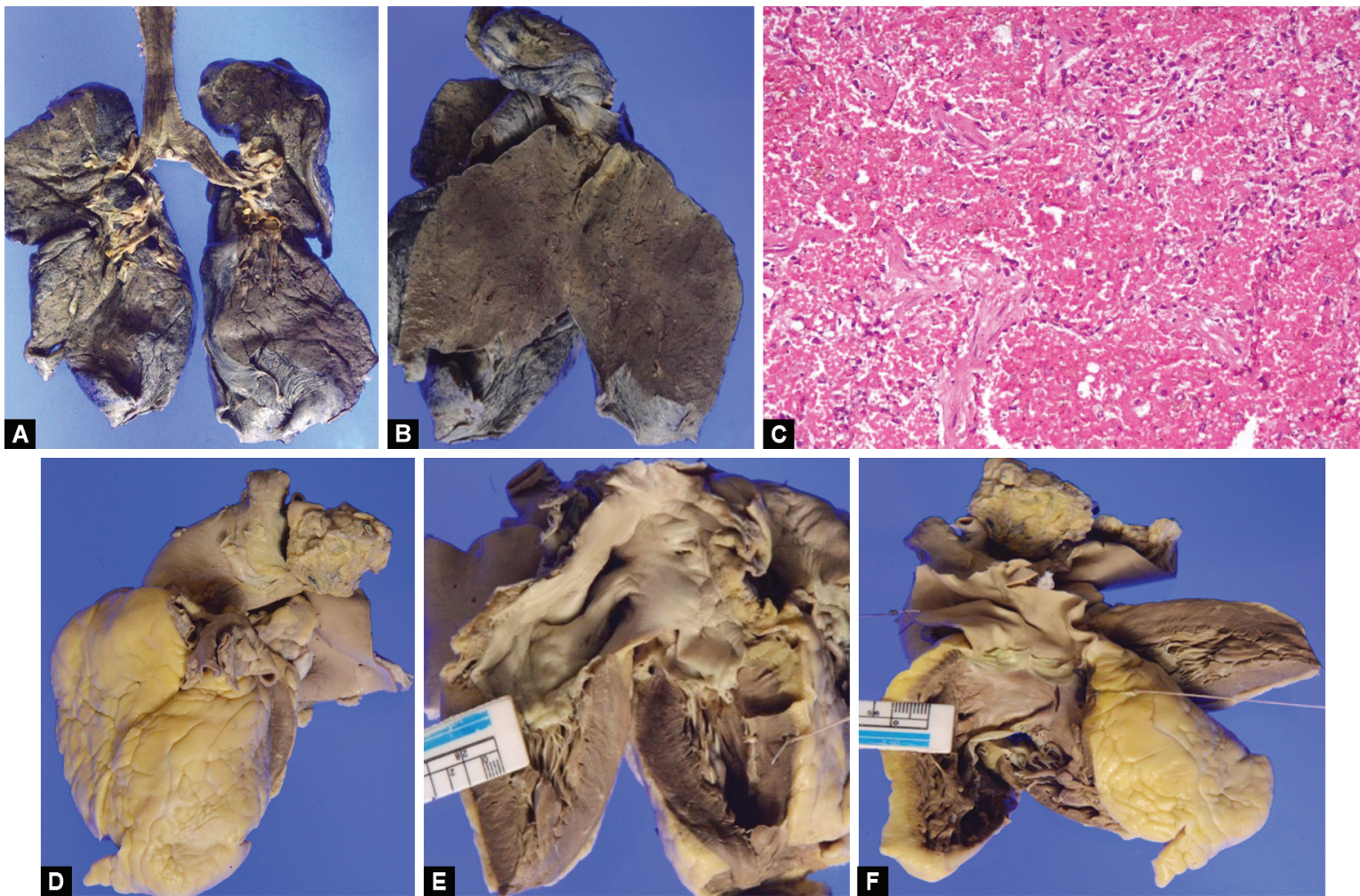

Figs 5A to F: (A) Organ complex comprising of bilateral lungs and trachea-bronchial tree. The trachea-bronchial tree did not show any aspirated material; (B) Cut surface of left lower lobe of lung showed hemorrhagic consolidation; (C) Diffuse alveolar hemorrhage was seen in the section taken from the area of hemorrhagic consolidation in left lower lobe of lung (hematoxylin and eosin 400X); (D) Heart was grossly unremarkable; (E) Right ventricular wall thickness was $0.4 \mathrm{~cm}$ and right outflow tract was unremarkable; (F) Left ventricular wall thickness was $1.4 \mathrm{~cm}$ and left inflow tract was unremarkable
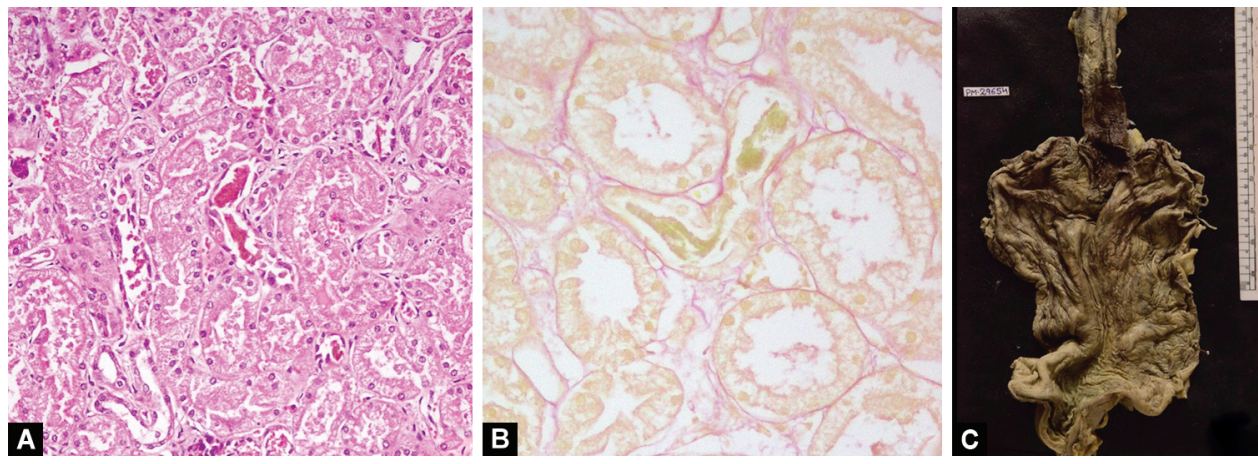

Figs 6A to C: (A) Kidney showed acute tubular necrosis with bile casts noted in the tubules (hematoxylin and eosin 400X); (B) Bile casts were highlighted on Fouchet's stain as green colored casts (Fouchet's stain, 400X); (C) Gross photograph showing esophagus and stomach showing erosion with areas of hemorrhage in distal esophagus and cardia of stomach

confirmed microscopically (Fig. 6). No infiltration by lymphoma cells was noted.

Gall bladder, pancreas, small and large intestines, skin and adrenal glands: all these organs were unremarkable both grossly and microscopically.

This patient had a $\mathrm{Bcl}-2$ positive DLBCL and poor prognostic significance of $\mathrm{bcl} 2$ positivity in DLBCL has been described by various authors. ${ }^{10-12}$

\section{Final Autopsy Diagnosis (PM29654)}

- Diffuse large B-cell lymphoma, NOS, non-GCB phenotype, and $\mathrm{BCl} 2$ expressor
- Lymphoma infiltration into liver, spleen, and bone marrow

- Diffuse alveolar hemorrhage

- Bile cast nephropathy

- Leiomyoma and uterus

\section{Final Discussion}

Prof Rakesh Kochar: Thank you. Dr Balamurugan, Please join me here.

Dr Gaurav: It was very nicely demonstrated pathology work up lymphoma. I am rather surprised to see such extensive extranodal involvement. There was no evidence of sepsis at all? 
Dr Balamurugan: Yes sir despite extensive sampling of lungs and other organs, there was no evidence of infection.

Prof Pankaj Malhotra: In our clinical practice, we do see such patients who present with massive tumor burden and give us no or limited time to treat.

Prof Sanjay Jain: It is surprising to see that despite so much tumor burden, LDH levels were not very high. Though we do not have uric acid levels, did you see any evidence of urate deposits which can be expected with so much turnover.

Dr Balamurugan: No, sir.

Prof Varinder Singh: So as expected from liver function tests, there was no hepatocytic loss and only lymphomatous infiltrate accounting for elevated bilirubin and raised alkaline phosphatase. Elevation of bilirubin must have been multifactorial.

Prof Rakesh Kochar: So today we witnessed nice demonstration of well worked up case of non-Hodgkin's lymphoma with massive tumor burden. Thank you everybody for the participation.

\section{Commentary}

High tumor load with involvement of multiple extranodal sites is a finding, which is usually noted in lymphomas which are double-hit or triple-hit and blastoid variant of mantle cell lymphoma. This was a rare case as it did not fall into any of the above three categories, and yet showed massive extranodal involvement.

Liver dysfunction can be multifactorial in cases of lymphoma as was in present case and rarely these diffuse large B cell lymphoma can present as acute liver failure. ${ }^{8,9}$

The adverse prognostic factors in this case included $A B C$ phenotype, Bcl2 positivity, and advance stage disease. ${ }^{10-12}$

Using gene expression profiling (GEP), DLBCL can be divided into cell of origin such as germinal center B-cell-like (GCB), activated $B$-cell-like $(A B C)$, and unclassified type. These groups have survival benefit independent of the IPI. GCB group has better overall survival than non-GCB group. ${ }^{13}$ Various studies report GCB subtype and non-GCB subtype range from $38 \%$ to $53 \%$ and $47 \%$ to $62 \%$, respectively. ${ }^{14-16}$ This tumor also had blastoid morphology at places, which placed double/triple-hit, lymphoblastic lymphoma and blastoid variant of mantle cell lymphoma as strong contender among the differentials and thus were excluded. c-myc and $\mathrm{Bcl} 2$ protein expression in DLBCL per se has poor prognostic value even without gene rearrangement. C-MYC, Bcl2, and C-MYC/BCL2 co-expression seen in DLBCL are 64\%, 50\%, and 34\%, respectively. ${ }^{17}$ $\mathrm{Hu}$ et al. stated that the MYC/BCL2 co-expression contributes to the inferior survival of $A B C$ phenotype of $D L B C L$ and there was increased C-MYC and BCL2 expression scores in non-GCB phenotype compared to GCB phenotype. ${ }^{17}$ Multivariate analysis performed showed significant poor overall survival and event-free survival in stages III, IV. ${ }^{14}$ Higher IPI score and stage still remains gold standard for assessment of prognosis, which is also demonstrated in this case.

\section{References}

1. Liu Y, Barta SK. Diffuse large B-cell lymphoma: 2019 update on diagnosis, risk stratification, and treatment. Am J Hematol 2019;94(5):604-616. DOI: 10.1002/ajh.25460.
2. Melchardt T, Troppan K, Weiss L, et al. A modified scoring of the NCCNIPI is more accurate in the elderly and is improved by albumin and ß2-microglobulin. Br J Haematol 2015;168(2):239-245. DOI: 10.1111/ bjh.13116.

3. LiS, Young KH, Medeiros LJ. Diffuse large B-cell lymphoma. Pathology 2018;50(1):74-87. DOI: 10.1016/j.pathol.2017.09.006.

4. Sehn LH, Berry B, Chhanabhai M, et al. The revised international prognostic index (R-IPI) is a better predictor of outcome than the standard IPI for patients with diffuse large B-cell lymphoma treated with R-CHOP. Blood 2007;109(5):1857-1861. DOI: 10.1182/blood-200608-038257.

5. Kubo K, Kimura N, Mabe K, et al. Acute liver failure associated with diffuse large B-cell lymphoma: an autopsy case report. Clin J Gastroenterol 2020. DOI: 10.1007/s12328-019-01091-6.

6. Fardet L, Galicier L, Lambotte O, et al. Development and validation of the HScore, a score for the diagnosis of reactive hemophagocytic syndrome. Arthritis Rheumatol 2014;66(9):2613-2620. DOI: 10.1002/ art.38690.

7. Singer $M$, Deutschman CS, Seymour CW, et al. The third international consensus definitions for sepsis and septic shock (sepsis-3). JAMA 2016;315(8):801-810. DOI: 10.1001/jama.2016.0287.

8. Kapuria D, Strasser K, Qasem A. Diffuse large B-cell lymphoma causing acute liver failure: a rare case of survival. BMJ Case Rep 2015;2015(1). DOI: 10.1136/bcr-2015-209328.

9. Shibata J, Kurahashi S, Naito T, et al. Diffuse large B cell lymphoma primarily presenting as acute liver failure in a surviving patient. J Community Hosp Intern Med Perspect 2019;9(2):135-139. DOI: 10.1080/20009666.2019.1583535.

10. Tsuyama N, Sakata S, Baba S, et al. BCL2 expression in DLBCL: reappraisal of immunohistochemistry with new criteria for therapeutic biomarker evaluation. Blood 2017;130(4):489-500. DOI: 10.1182/blood-2016-12-759621.

11. Li L, Li $Y$, Que $X$, et al. Prognostic significances of overexpression MYC and/or BCL2 in R-CHOP-treated diffuse large B-cell lymphoma: a systematic review and meta-analysis. Sci Rep 2018;8(1):6267. DOI: 10.1038/s41598-018-24631-5.

12. Tang $Y L$, Zhou $Y$, Cheng $L L$, et al. BCL2/ki-67 index predict survival in germinal center B-cell-like diffuse large B-cell lymphoma. Oncol Lett 2017;14(3):3767-3773. DOI: 10.3892/ol.2017.6577.

13. Alizadeh AA, Eisen MB, Davis RE, et al. Distinct types of diffuse large B-cell lymphoma identified by gene expression profiling. Nature 2000;403(6769):503-511. DOI: 10.1038/35000501.

14. Coutinho R, Clear AJ, Owen A, et al. Poor concordance among nine immunohistochemistry classifiers of cell-of-origin for diffuse large b-cell lymphoma: Implications for therapeutic strategies. Clin Cancer Res 2013;19(24):6686-6695. DOI: 10.1158/1078-0432.CCR-131482.

15. Dwivedi A, Mehta A, Solanki P. Evaluation of immunohistochemical subtypes in diffuse large B-cell lymphoma and its impact on survival. Indian J Pathol Microbiol 2015;58(4):453-458. DOI: 10.4103/03774929.168886.

16. Gutiérrez-García G, Cardesa-Salzmann T, Climent F, et al. Geneexpression profiling and not immunophenotypic algorithms predicts prognosis in patients with diffuse large B-cell lymphoma treated with immunochemotherapy. Blood 2011;117(18):4836-4843. DOI: 10.1182/ blood-2010-12-322362.

17. Hu S, Xu-Monette ZY, Wu L, et al. MYC/BCL2 protein co-expression defines a unique subset of aggressive lymphoma and contributes to the inferior prognosis of activated B-cell subtype of diffuse large B-cell lymphoma: a report from the international DLBCL rituximabCHOP consortium program s. Clin Lymphoma, Myeloma Leuk 2013;13(20):S382-S383. DOI: 10.1016/j.clml.2013.07.095. 